\title{
Dermatomal Somatosensory Evoked Potentials: An Indicator of Improvement of Breast Sensibility after Reduction Mammaplasty
}

\author{
Mohammad Abu-Hegazy1", Wael Gabr ${ }^{1}$, Hossam El-Din A. Ismail2, Ahmad A. Khali12 \\ ${ }^{1}$ Department of Neurology, Mansoura University, Mansoura, Egypt \\ ${ }^{2}$ Department of Plastic and Reconstructive Surgery, Mansoura University, Mansoura, Egypt \\ Email: "mhegazy@mans.edu.eg
}

Received 21 June 2016; accepted 28 August 2016; published 31 August 2016

Copyright (C) 2016 by authors and Scientific Research Publishing Inc.

This work is licensed under the Creative Commons Attribution International License (CC BY). http://creativecommons.org/licenses/by/4.0/

(c) (i) Open Access

\section{Abstract}

Introduction: Many women think about reduction mammaplasty for different reasons. The effect of surgery on the beast sensibility is one of the greatest concerns after reconstructive reductive breast surgery through its affect on patient's social life and psychological health. The dermatomal somatosensory evoked potential (D-SEP) is a new method to quantitatively evaluate breast sensibility. Patients and Methods: Twenty-five women enrolled in this study presenting with breast enlargement, underwent mammary reduction by using the inferior pyramidal breast reduction technique using the same operative technique described by Robbins with some modifications. All D-SEP amplitudes and latencies were calculated preoperatively and then were reassessed six and twelve months post-surgery in each breast. Result: The results revealed that there is a significant difference in the D-SEP latency pre- and post-operatively. The statistically significant decrease in latency and the breast size demonstrated indicates that the sensibility improved after breast reduction surgery both at six and twelve months. There is also a significant increase in the D-SEP amplitude pre- and post-operatively. The negative and statistically significant increase in amplitude with the decrease in breast size demonstrated indicates that the sensibility improved after breast reduction surgery both at six and twelve months. Conclusion: This study concluded that breast sensibility will improve after breast reduction as indicated by significant reduction of D-SEP latencies and increase of its amplitudes. Our results confirm an inverse relationship between breast size and sensibility, with improvement in sensibility after breast reduction.

*Corresponding author.

How to cite this paper: Abu-Hegazy, M., Gabr, W., Ismail, H.A. and Khalil, A.A. (2016) Dermatomal Somatosensory Evoked Potentials: An Indicator of Improvement of Breast Sensibility after Reduction Mammaplasty. Neuroscience and Medicine, 7, 106-113. http://dx.doi.org/10.4236/nm.2016.73012 


\section{Keywords}

\section{Breast Sensibility, Reduction Mammoplasty, Dermatomal Somatosensory Evoked Potentials}

\section{Introduction}

Ideal, natural and symmetric breast shape is the aim for mammaplasty [1], but breast sensibility is an important issue for each woman which can affect the female sexual activity, social life and psychological health [2].

Tayrish et al. [3] found that breast sensation affected by breast size and ptosis in healthy females and revealed that increase in the breast size significantly attenuates the breast sensitivity. They attributed this to breast cutaneous nerves prolonged stretch, traction and/or injury. Changes in the breast sensibility after reduction mammaplasty remain a controversial subject [4].

Many women think about reduction mammaplasty for different reasons, but one of the greatest concerns among patients presenting for reconstructive reductive breast surgery is the subsequent effect of surgery on the beast sensibility which can affect the patient social life and psychological health [2]. Sensibility recovery is very important to enhance neo-breast body image integration and normal sexual activity restoration [1].

The breast sensibility including pain, pressure, temperature, touch, vibration, two points discrimination and the erogenous sensation, is a subjective condition that is difficult to be evaluated [5]. To evaluate breast sensibility after surgery, many tests were used including light touch using cotton wool, pin-prick test, two-point discrimination and monofilament test using Semmes-Weinstein monofilament. All these tests are subjective tests [2], and quantitative sensibility studies are needed to compare sensation before and after reduction mammaplasty to give a clear answer for effect of breast reduction surgery on breast sensibility [4].

The dermatomal somatosensory evoked potential (D-SEP) is a new method to quantitatively evaluate breast sensibility [6]. In 1875, Richard Caton discovered evoked potentials [7]. Evoked potentials are the electrical activity produced by groups of neurons within the spinal cord, thalamus, brain stem or brain in response to an external stimulus mainly by visual, auditory, or somatosensory stimulus [8]. In 2004, Del Vecchyo and coworkers [6] used D-SEPs to quantitatively evaluate in breast sensitivity after mammaplasty. In the current study the breast sensibility was evaluated preoperative and at 6 and 12 months postoperatively after breast reduction using the D-SEPs.

\section{Patients and Methods}

The breast sensory testing protocol and consent procedures were approved by the scientific committee at Mansoura University Hospital.

Twenty-five women with breast gigantomastia presented to plastic surgery clinic who underwent mammary reduction in excess of $700 \mathrm{~g}$ each side (range, $700-2000 \mathrm{~g}$ ) by using the inferior pyramidal breast reduction technique at Mansoura University Hospital between January 2013 to January 2015 are enrolled in this study. They were referred by the plastic surgeon to neurophysiology unit in Mansoura University Hospital for sensory examination.

All participants provided written informed consent to undergo breast sensory sensibility testing, which was done one night before surgery using dermatomal somatosensory evoked potential, and repeated again 6 and 12 months post-operatively.

The average age of patients was 36.1 years. Breast size among participants ranged from 36DD to 46EE (10 females had a cup sized DD, 12 had cup sized E and only 3 had a cup sized EE).

The exclusion criteria included:

Patients with diabetes mellitus, hypertension, thyroid diseases, alcoholism, collagen vascular disease, pernicious anemia, neurologic conditions that may alter the nervous system response and previous mammary surgery.

\subsection{Surgical Procedures}

Preoperative marking was done while the patient was standing according to Wise pattern inferior pedicle technique described by Robbins [9]. The sternal notch, inframammary fold (IMF), midline and breast meridian were 
marked as a line drawn from the mid portion of the clavicle to the nipple. If this line is greater than 10 to $12 \mathrm{~cm}$ from the midline, then it should be relocated medially. The new nipple areola location was marked just at or slightly below the IMF with a diameter of approximately $4 \mathrm{~cm}$. The distances from the midclavicular point to the position of the new nipples at the breast meridian ranging from $23-25 \mathrm{~cm}$. The distances from the sternal notch to the position of the new nipples can be adjusted based on patient height, magnitude of the planned reduction, and surgeon preference. The vertical limbs were drawn obliquely down from the new nipple center mark ranging from $8-9 \mathrm{~cm}$. The vertical limbs form an equilateral triangle. The base of the triangle should be approximately 9 - $11 \mathrm{~cm}$ wide, depending on the degree of reduction desired. A wide pedicle base $(11-15 \mathrm{~cm})$ is preferred. Because lateral, medial, and superior glandular resection is usually performed in the mastectomy plane, the main bulk of the remaining breast is made up of pedicle tissue. This means that the lateral and medial flaps are relatively thin also to obtain tension free reduction.

We used, with some modifications, the operative technique described by Robbins. During excision of the breast fat and parenchyma, care was taken to preserve at least as much attachment of the pedicle to the chest in its vertical dimension as its width and also by fashioning the pedicle as a pyramid with its base on the chest wall (Figure 1).

The addition of pedicle suspension, by suturing the dermis at the upper part of the pedicle and about $4 \mathrm{~cm}$ above the IMF with an interrupted 3/0 polypropylene suture to the pectoral fascia was our modifications to the technique. The wounds and the areola were closed into two layers using interrupted subcutaneous $3 / 0$ vicryl and a 4/0 polypropylene intracuticular suture. Closed suction drains and stri strips were applied to inverted T-incision and the areola the absorbent dressings and Bravoplast are used routinely. Also soft bra for 3 months.

\subsection{Evoked Somatosensory Evaluation}

Breast sensibility was evaluated in all patients (50 breasts in 25 patients) by the same examiner, using the D-SEPs.

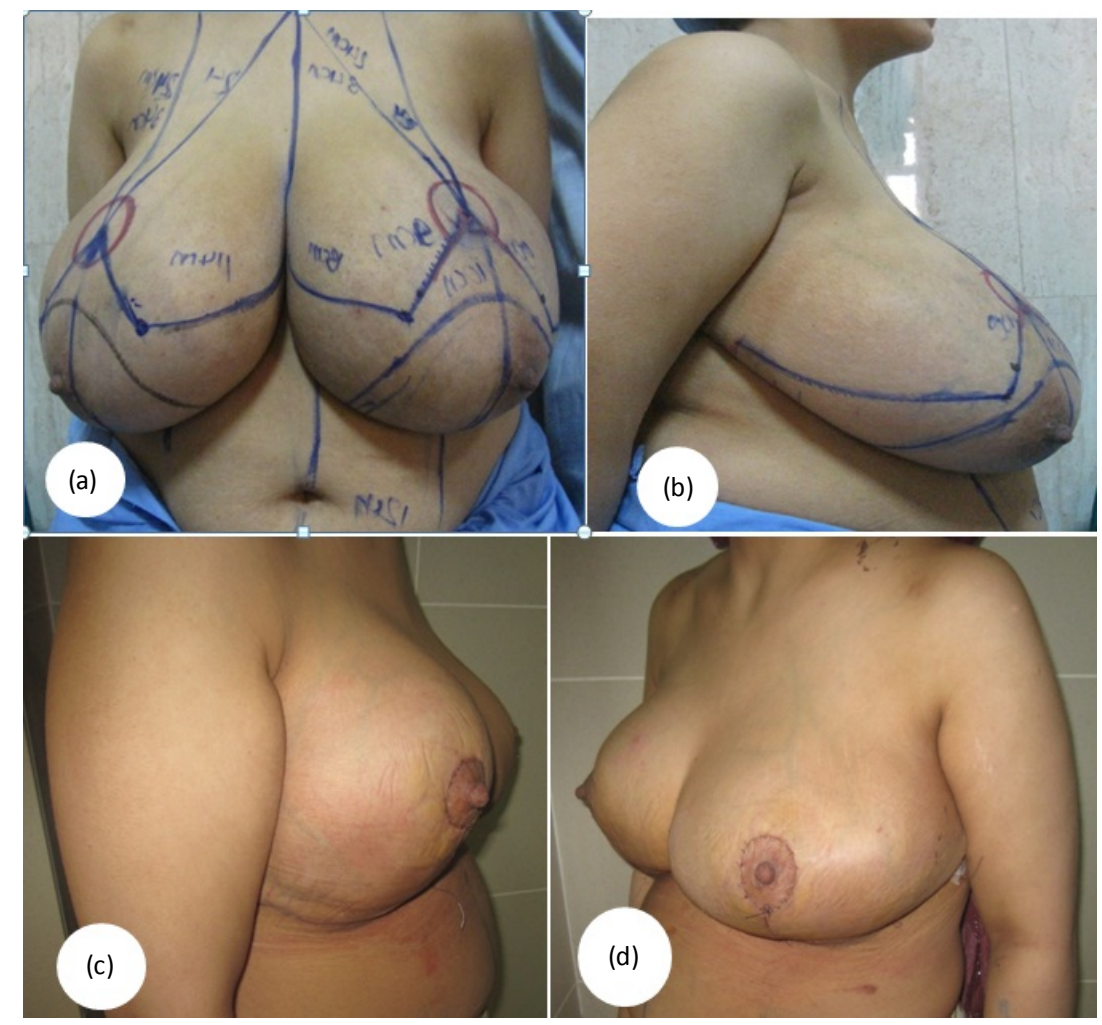

Figure 1. A 37-year-old patient, subjected to reduction mamaplasty using the inferior pyramidal technique. (a) Preoperative marking; (b) Right lateral view; (c) Early post operative rtight lateral view; (d) The same patient early post operative left lateral view. 
D-SEP examination was done with the patient lying supine in comfortable warm semidarkened room uing NIHON KOHDEN evoked response recorder model MEP-5200. The patients remain awake throughout the whole examination with eyes opened (Figure 2).

Dermatomal stimulation was performed to the superior quadrant, the nipple-areola complex and the inferior quadrant that correspond to T3, T4, and T5 dermatomes respectively, according to dermatomal SEP maps [10].

To record the cortical D-SEPs, disc electrodes were positioned over the scalp in a frontal plane that was $2 \mathrm{~cm}$ posterior to the interaural-Cz plane (10 - 20 system), at C1' (between C3 and P3) and C2' (between C4 and P4) (10 percent of the interaural plane lateral to the midline) contralateral to the stimulating side. All electrodes were referred Fpz. A ground electrode was placed on the ipsilateral forearm.

Stimulation was done using bipolar stimulating surface electrodes (9-mm-diameter discs embedded in a plastic band separated by $3 \mathrm{~cm}$ ), with the cathode directed proximally.

The stimulus intensity used was adjusted 3 times the sensory threshold, without exceeding the pain threshold. the pulse duration was $0.2 \mathrm{msec}$ and the repetition rate was 2 Hertz. Amplification with high cut filter $100 \mathrm{~Hz}$ and low cut $2 \mathrm{~Hz}$.

Repetitive electrical stimulations (average count of 200) were delivered to the skin overlying the T3, T4 and T5 dermatomes on each breast. For each stimulation site, all traces were examined and the trace yielding the maximum amplitude of the initial component (N1-P1) was selected for data analysis.

All amplitudes (in millivolt) and latencies (in millisecond) were calculated. The latency and amplitude values obtained from patients after breast reduction were compared with their own preoperative values.

Statistical computations were done using SPSS version 16 (Chicago, IL, USA). Measurements to determine breast sensation, at three different time points (preoperatively, at 6 and 12 months postoperatively) were analyzed using One-Way ANOVA. Statistical significance was predefined as $\mathrm{P} \leq 0.05$. The data are described in terms of the means and standard errors of the means.

\section{Results}

Twenty-five consecutive female patients who underwent reduction mammaplasty by inferior pyramidal technique were enrolled in this study.

Ages of participants ranged from 25 to 46 years, with a mean age of $36.1 \pm 7.1$ years. Fortunately, only minor complications occurred as wound dehiscence, hypertrophic scars, and local wound healing problems, which were treated medically. No major complications occurred. There was partial areolar necrosis (two cases), healed without surgical intervention. Healing was uneventful, and all the patients were highly satisfied with the outcome. The dermatol somatosensory evoked potential latencies and amplitudes were measured preoperatively and then were reassessed six and twelve months postoperatively in each breast.

Table 1 revealed a significant reduction in the D-SEP mean latency 6 months postoperatively with further reduction at 12 months, indicating improvement of breast sensibility. In other words D-SEP latency is directly proportionate to the breast dermatomal size.

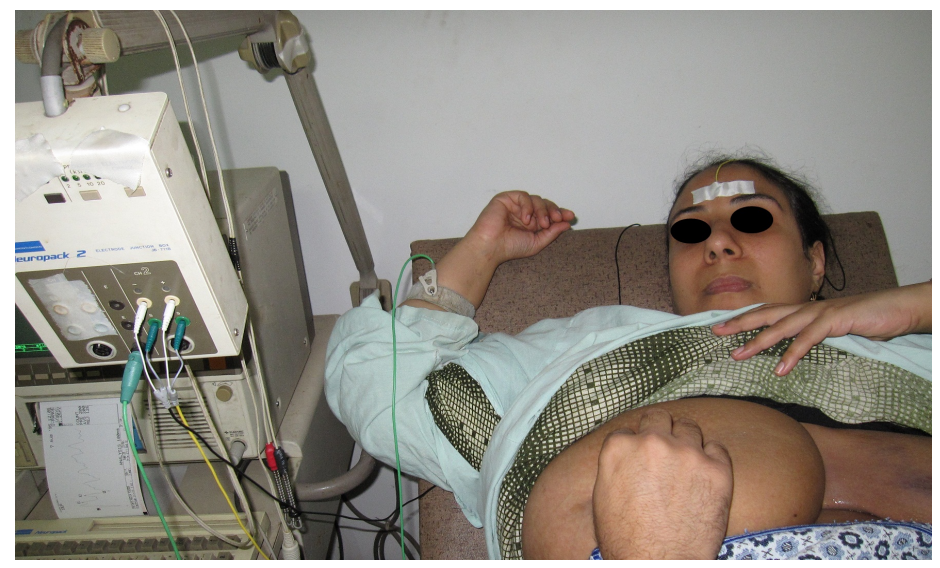

Figure 2. A 35-year-old patient, subjected to preoperative assessment by D-SEP. 
Table 1. Pre- and post-operative D-SEP latency in different bilateral breast quadrants.

\begin{tabular}{cccccccc}
\hline & & \multicolumn{2}{c}{ Right breast } & \multicolumn{2}{c}{ Left breast } & \multicolumn{2}{c}{ Left \& right breast } \\
\cline { 3 - 8 } & & Mean \pm SD & P & Mean \pm SD & P & Mean \pm SD & P \\
\hline \multirow{2}{*}{ Superior quadrant } & Preoperative & $29.33 \pm 2.03$ & & $29.17 \pm 2.07$ & & $58.50 \pm 3.65$ \\
& $\mathbf{6}$ M postoperative & $28.11 \pm 2.09^{*}$ & 0.043 & $26.84 \pm 1.32^{*}$ & 0.045 & $57.39 \pm 1.25^{*}$ & 0.044 \\
& $\mathbf{1 2}$ M postoperative & $27.06 \pm 1.62^{*}$ & 0.031 & $26.34 \pm 1.08^{*}$ & 0.028 & $53.40 \pm 2.61^{*}$ & 0.029 \\
\hline & Preoperative & $29.78 \pm 2.32$ & & $28.79 \pm 1.32$ & & $59.32 \pm 1.23$ \\
Nipple-areola complex & $\mathbf{6}$ M postoperative & $28.72 \pm 2.37^{*}$ & 0.039 & $27.87 \pm 1.02^{*}$ & 0.049 & $56.60 \pm 3.23^{*}$ & 0.044 \\
& $\mathbf{1 2}$ M postoperative & $27.75 \pm 1.82^{*}$ & 0.028 & $26.74 \pm .79^{*}$ & 0.040 & $54.49 \pm 2.03^{*}$ & 0.034 \\
\hline & Preoperative & $30.13 \pm 2.10$ & & $29.90 \pm 2.25$ & & $60.02 \pm 4.31$ & \\
Inferior quadrant & $\mathbf{6}$ M postoperative & $28.93 \pm 1.62^{*}$ & 0.028 & $28.87 \pm 2.17^{*}$ & 0.047 & $58.99 \pm 4.23^{*}$ & 0.038 \\
& $\mathbf{1 2}$ M postoperative & $27.80 \pm 1.25^{*}$ & 0.019 & $27.55 \pm 1.65^{*}$ & 0.028 & $55.35 \pm 2.74^{*}$ & 0.024 \\
\hline
\end{tabular}

*Significant difference of mean when compared to preoperative mean ( $\mathrm{P}$ value $<0.050)$.

Investigating D-SEP amplitude in different dermatomes showed no significant amplitude changes in the superior quadrant at different post-operative study time points. There was also no significant amplitude change in nipple areola complex at different post-operative study time points. Surprisingly there was significant increase in D-SEP amplitude in the lower quadrant at 12 -month postoperatively in both sides $(\mathrm{P}=0.019,0.009,0.014)$ for right, left, and both breasts respectively (Table 2).

Figure 3 and Figure 4 is a pre- and post-reduction mammoplasty D-SEP in a 31-year-old lady.

\section{Discussion}

Breast sensibility changes after surgical breast reduction remain a controversial subject and most previous studies use subjective methods in assessment [4], Objective data on breast sensation are scarce [11].

Female breast innervated by the lateral and anterior cutaneous branches of the intercostal nerves from the second to the sixth nerves which are scarified during breast mammaplasty [12]. Breast re-innervation is not done by most surgeons; they mainly depend on the regeneration of nerves from the adjacent residual post-mastectomy skin [13].

The breast sensibility including; pain, pressure, temperature, touch, vibration, two points discrimination and the erogenous sensation, is a subjective condition that is difficult to be evaluated [5], Many tests were used in literatures to evaluate breast sensibility after surgery including light touch using cotton wool, pin-prick test, two-point discrimination and monofilament test using Semmes-Weinstein monofilament, all these tests are subjective tests [2], and a quantitative sensibility studies are needed to compare sensation before and after reduction mammaplasty to give a clear answer for effect of breast reduction surgery on breast sensibility [4].

Dermatomal somatosensory evoked potentials involve recording cerebral evoked responses from cutaneous electrical stimulation of known dermatomal areas providing a pure sensory input to any level of the spinal cord up to the cerebral cortex [14].

D-SEPs can be conducted by electrical stimulation of peripheral nerves. A single skin dermatome stimulation elicits a centrally recorded response that should reflect impulses traversing a single root. At the root level, slowing or blocking the impulse become identifiable [15]. A normal dermatomal somatosensory evoked potential response at the scalp indicates intact segmental level conduction of the stimulated dermatome as well as along the somatosensory pathway till the cerebral cortex. An abnormal response suggests segmental level impairment [16].

Del Vecchyo and co-workers [6] were the first researchers to use D-SEPs, as a method of evaluating sensibility before and after breast reduction mammpoplasty. They have detected normative values in small-breasted women and have determined differences in sensibility according to breast size and after breast reduction. Their results confirmed that there was an inverse relationship between sensibility and breast size and improvement in sensibility after breast reduction. Their result also support a possible overlap between adjacent brest skin dermatomes. 


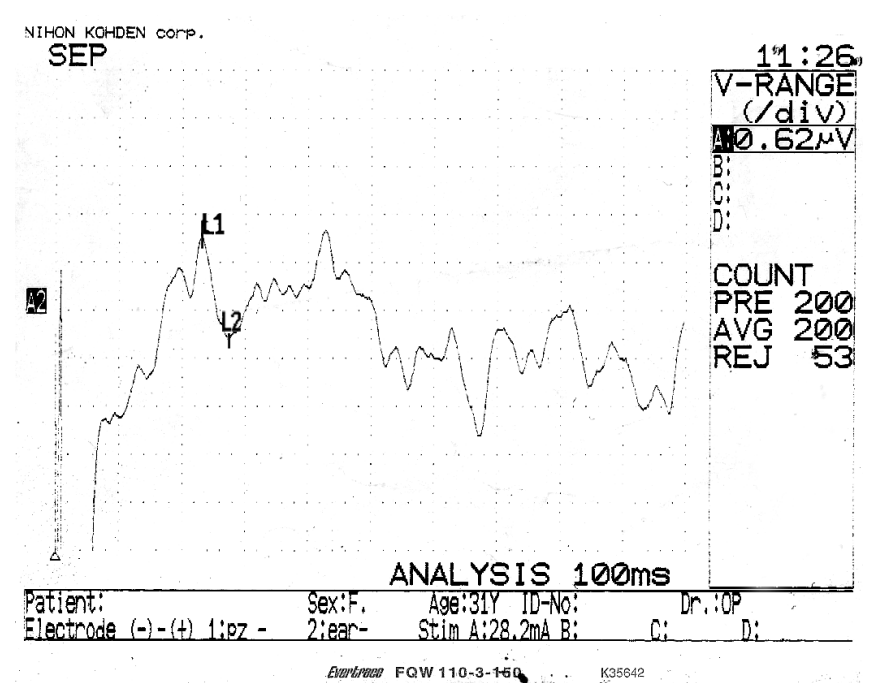

Figure 3. Pre-operative D-SEP in a 32-year-old patient.

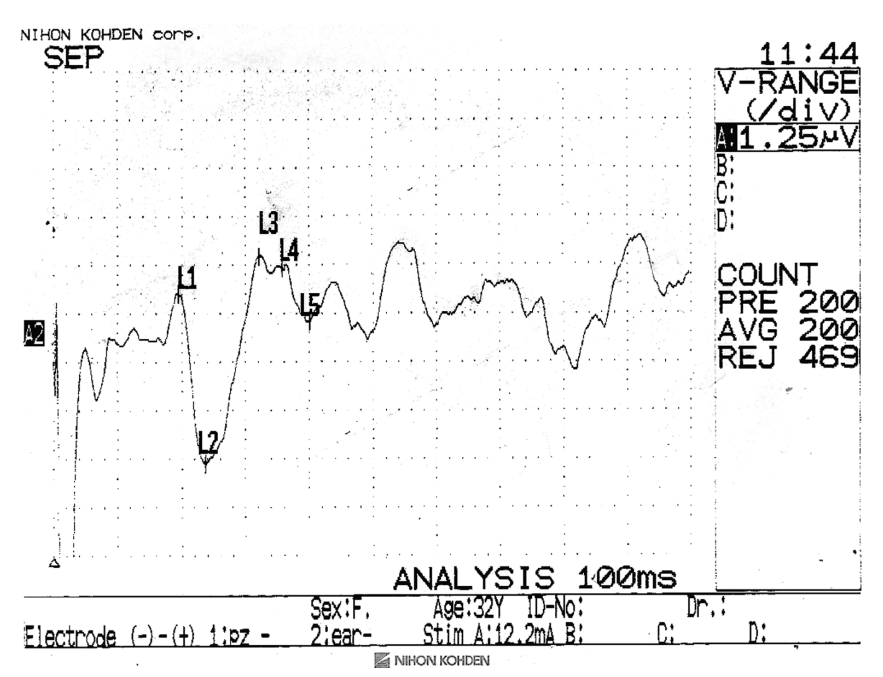

Figure 4. Post-operative D-SEP in a 32-year-old patient.

Table 2. Pre- and post-operative D-SEP amplitude in different bilateral breast quadrants.

\begin{tabular}{cccccccc}
\hline & & \multicolumn{2}{c}{ Right breast } & \multicolumn{2}{c}{ Left breast } & \multicolumn{2}{c}{ Left \& right breast } \\
\cline { 3 - 7 } & & Mean \pm SD & P & Mean \pm SD & P & Mean \pm SD & P \\
\hline \multirow{2}{*}{ Superior quadrant } & Preoperative & $0.444 \pm 0.096$ & & $0.463 \pm 0.092$ & & $0.91 \pm 0.19$ \\
& $\mathbf{6}$ M postoperative & $0.553 \pm 0.072$ & 0.061 & $0.586 \pm 0.074$ & 0.059 & $1.14 \pm 0.15$ & 0.060 \\
& $\mathbf{1 2}$ M postoperative & $0.567 \pm 0.063^{*}$ & 0.049 & $0.604 \pm 0.064^{*}$ & 0.050 & $1.17 \pm 0.13^{*}$ & 0.049 \\
\hline $\begin{array}{c}\text { Nipple-areola } \\
\text { complex }\end{array}$ & Preoperative & $0.524 \pm 0.099$ & & $0.433 \pm 0.090$ & & $0.96 \pm 0.18$ \\
& $\mathbf{6}$ M postoperative & $0.530 \pm 0.068$ & 0.071 & $0.495 \pm 0.106$ & 0.059 & $1.03 \pm 0.17$ & 0.065 \\
& $\mathbf{1 2}$ M postoperative & $0.551 \pm 0.060$ & 0.062 & $0.510 \pm 0.099$ & 0.051 & $1.06 \pm 0.16$ & 0.056 \\
\hline Inferior quadrant & $\mathbf{6}$ M postoperative & $0.510 \pm 0.068$ & 0.061 & $0.486 \pm 0.066^{*}$ & 0.049 & $1.08 \pm 0.13^{*}$ & 0.055 \\
& $\mathbf{1 2}$ M postoperative & $0.550 \pm 0.062^{*}$ & 0.019 & $0.562 \pm 0.059^{*}$ & 0.009 & $1.11 \pm 0.12^{*}$ & 0.014 \\
\hline
\end{tabular}

*Significant difference of mean when compared to preoperative mean ( $\mathrm{P}$ value $<0.050)$. 
In the current study 25 consecutive female patients who underwent reduction mammaplasty by inferior pyramidal technique were enrolled in this study. The latencies and amplitudes of the dermatol somatosensory evoked potential were measured preoperatively and then were reassessed six and twelve months postoperatively in each breast. Ages of participants ranged from 25 to 46 years, with a mean age of years. The average age of participants was $36.1 \pm 7.1$ years. Breast size among participants ranged from 36DD to 46EE (10 females had a cup sized DD, 12 had cup sized E and only 3 had a cup sized EE).

Our result revealed a significant improvement of breast sensibility as indicated by significant reduction of D-SEPs terminal latencies and significant increase in their amplitudes after breast reduction, which is prominent after six months postoperatively and continue to improve over the next months as indicated by the measurement obtained at one year postoperatively. This improvement may be explained by the decrease in the breast size after reduction mammoplasty with subsequent reduction of the breast ptosis and abolishment of the stretch traction/injury to the cutaneous nerves of the breast [17], while our results concluded improvement of the breast sensibility, Hamdi and his colleagues at 2001 found that sensibility may be decreased [18], but most of the studies done in this field of research concluded improvement in sensibility, augmenting our results [11] [16] [19].

\section{Conclusion}

This study concluded that breast sensibility will improve after breast reduction as indicated by significant reduction of D-SEP latencies and increase of its amplitudes. Our results confirm an inverse relationship between breast size and sensibility, with improvement in sensibility after breast reduction.

\section{References}

[1] Santanelli, F., Longo, B., Angelini, M., Laporta, R. and Paolini, G. (2011) Prospective Computerized Analyses of Sensibility in Breast Reconstruction with Non-Reinnervated DIEP Flap. Plastic and Reconstructive Surgery, 127, 1790 1795. http://dx.doi.org/10.1097/PRS.0b013e31820cf1c6

[2] Mofid, M.M., Dellon, A.L., Elias, J.J. and Nahabedian, M.Y. (2002) Quantitation of Breast Sensibility Following Reduction Mammaplasty: A Comparison of Inferior and Medial Pedicle Techniques. Plastic and Reconstructive Surgery, 109, 2283-2288. http://dx.doi.org/10.1097/00006534-200206000-00018

[3] Tairych, G.V., Kuzbari, R., Rigel, S., Todoroff, B.P., Schneider, B. and Deutinger, M. (1998) Normal Cutaneous Sensibility of the Breast. Plastic and Reconstructive Surgery, 102, 701-704. http://dx.doi.org/10.1097/00006534-199809010-00013

[4] Ferreira, M.C., Costa, M.P., Cunha, M.S., Sakae, E. and Fels, K.W. (2003) Sensibility of the Breast after Reduction Mammaplasty. Annals of Plastic Surgery, 51, 1-5. http://dx.doi.org/10.1097/01.SAP.0000054190.76311.1A

[5] Prado, A., Andrades, P., Benitez, S. and Parada, F. (2008) Areola-Nipple Perception Threshold to Faradic Electricity: A New Measure of Sensibility of the Breasts. Aesthetic Plastic Surgery, 32, 748-752. http://dx.doi.org/10.1007/s00266-008-9148-4

[6] DelVecchyo, C., Caloca Jr., J., Caloca, J. and Gomez-Jauregui, J. (2004) Evaluation of Breast Sensibility Using Dermatomal Somatosensory Evoked Potentials. Plastic and Reconstructive Surgery, 113, 1975-1983. http://dx.doi.org/10.1097/01.PRS.0000122210.12819.B8

[7] Shagass, C. (1976) Evoked Brain Potential in Man. In: Grenell, R. and Sabit, G., Eds., Biological Foundations of Psychiatry, Raven Press, New York, 199-253.

[8] Chiappa, K.H., Martin, J.B. and Young, R.R. (1987) Diagnostic Methods in Neurology: Disorders of the Central Nervous System. In: Martin, J.B., Ed., Harrison’s Principles of Internal Medicine, McGraw-Hill, Hamburg, $1913-1921$.

[9] Robbins, T.H. (1984) Inferior Pedicle Breast Reduction Technique. Plastic and Reconstructive Surgery, $73,325$. http://dx.doi.org/10.1097/00006534-198402000-00044

[10] Downs, M.B. and Laporte, C. (2011) Conflicting Dermatome Maps: Educational and Clinical Implications. Journal of Orthopaedic \& Sports Physical Therapy, 41, 427-434. http://dx.doi.org/10.2519/jospt.2011.3506

[11] Gonzalez, F., Brown, F.E., Gold, M.E., Walton, R.L. and Shafer, B. (1993) Preoperative and Postoperative NippleAreola Sensibility in Patients Undergoing Reduction Mammaplasty. Plastic and Reconstructive Surgery, 92, 809-814. http://dx.doi.org/10.1097/00006534-199392050-00005

[12] Sarhadi, N.S., Shaw, D.J., Lee, F.D. and Soutar, D.S. (1996) An Anatomical Study of the Nerve Supply of the Breast, including the Nipple and Areola. British Journal of Plastic Surgery, 49, 156-164. http://dx.doi.org/10.1016/S0007-1226(96)90218-0

[13] Spiegel, A.J., Menn, Z.K., Eldor, L., Kaufman, Y. and Dellon, A.L. (2013) Breast Reinnervation: DIEP Neurotization 
Using the Third Anterior Intercostal Nerve. Plastic and Reconstructive Surgery Global Open, 1, e72. http://dx.doi.org/10.1097/gox.0000000000000008

[14] (1997) Assessment: Dermatomal Somatosensory Evoked Potentials. Report of the American Academy of Neurology’s Therapeutics and Technology Assessments Subcommittee. Neurology, 49, 1127-1130. http://dx.doi.org/10.1212/WNL.49.4.1127

[15] Rodríguez, A.A., Kanis, L., Rodríguez, A.A. and Lane, D. (1987) Somatosensory Evoked Potentials from Dermatomal Stimulation as an Indicator of L5 and S1 Radiculopathy. Archives of Physical Medicine and Rehabilitation, 68, 336338.

[16] Slimp, J.C., Rubner, D.E., Snowden, M.L. and Stolov, W.C. (1992) Dermatomal Somatosensory Evoked Potentials: Cervical, Thoracic, and Lumbosacral Levels. Electroencephalography and Clinical Neurophysiology, 84, 55-70. http://dx.doi.org/10.1016/0168-5597(92)90068-M

[17] Tairych, G.V., Kuzbari, R., Rigel, S., Todoroff, B.P., Schneider, B. and Deutinger, M. (1998) Normal Cutaneous Sensibility of the Breast. Plastic and Reconstructive Surgery, 102, 701-704. http://dx.doi.org/10.1097/00006534-199809010-00013

[18] Hamdi, M., Greuse, M., De, M.A. and Webster, M.H. (2001) A Prospective Quantitative Comparison of Breast Sensation after Superior and Inferior Pedicle Mammaplasty. British Journal of Plastic Surgery, 54, 39-42. http://dx.doi.org/10.1054/bjps.2000.3456

[19] Temple, C.L. and Hurst, L.N. (1999) Reduction Mammaplasty Improves Breast Sensibility. Plastic and Reconstructive Surgery, 104, 72-76. http://dx.doi.org/10.1097/00006534-199907000-00010

\section{Submit or recommend next manuscript to SCIRP and we will provide best service for you:}

Accepting pre-submission inquiries through Email, Facebook, LinkedIn, Twitter, etc. A wide selection of journals (inclusive of 9 subjects, more than 200 journals)

Providing 24-hour high-quality service

User-friendly online submission system

Fair and swift peer-review system

Efficient typesetting and proofreading procedure

Display of the result of downloads and visits, as well as the number of cited articles

Maximum dissemination of your research work

Submit your manuscript at: http://papersubmission.scirp.org/ 